\title{
Intrapancreatic accessory spleen: An imaging enigma
}

\author{
Saksham P Yadav, Shivam D Kotak, Sachin Kumar, Vishnu S Pujari, Akhila Chandrasekaran \\ Department of Radiology, Kokilaben Dhirubhai Ambani Hospital and Medical Research Institute, Mumbai, Maharashtra, India
}

Correspondence: Dr. Sachin Kumar, Department of Radiology, Kokilaben Dhirubhai Ambani Hospital and Medical Research Institute, E-mail: sachinkumar89@gmail.com

\begin{abstract}
The pancreatic tail is an uncommon location for the accessory spleen. Although it is a benign entity, it can mimic and get misdiagnosed as a pancreatic tumor which can lead to unnecessary biopsy and surgery. Here, we present a case who was detected to have a tail of pancreas mass. On CT and MRI, it showed similar density, signal intensity, and matching enhancement pattern with the orthotopic spleen. The ADC value of the mass was found to be similar to that of the spleen and significantly less than that of normal pancreas. A diagnosis of intrapancreatic accessory spleen was hence made and the patient was followed up after 6 months on MRI. No change in lesion morphology and size was noted. Thus, intrapancreatic accessory spleen should be kept in mind as a differential diagnosis while reviewing a case with pancreatic mass.
\end{abstract}

Key words: Accessory spleen; computed tomography; intrapancreatic accessory spleen; magnetic resonance imaging; pancreas; spleen

\section{Introduction}

An accessory spleen is a benign and relatively common congenital entity resulting from failure of fusion of the splenic anlage during embryologic splenic development. ${ }^{[1]}$ The incidence is about $10-30 \%{ }^{[2]}$ with splenic hilum (80\%) being the most common site followed by the pancreatic tail $(17 \%) .{ }^{[3]}$ The intrapancreatic accessory spleen (IPAS) poses a diagnostic challenge on imaging and often gets misdiagnosed as pancreatic tumor, ${ }^{[4]}$ which can lead to unnecessary surgery or biopsy. Thus, accurate diagnosis of IPAS might save the patient from unwarranted intervention.

\section{Case History}

A 55-year-old male presented to our institution with complaint of epigastric pain for 10-15 days which was

\begin{tabular}{|l|l|}
\hline \multicolumn{2}{|c|}{ Access this article online } \\
\hline Quick Response Code: & \\
\hline & Website: \\
\hline & www.ijri.org \\
\cline { 2 - 3 } & DOI: \\
\hline
\end{tabular}

intermittent and dull aching. On the physical examination, the abdomen was non-tender. An ultrasound examination done elsewhere suggested a well-defined mass lesion at the tail of the pancreas. The serum amylase and lipase levels, complete blood count, and differential white blood cell count were normal. Contrast-enhanced CT scan of abdomen was done at our institute, which showed a well-defined, enhancing mass lesion in the pancreatic tail. The enhancement of mass matched to that of the spleen in arterial as well as venous phases [Figure 1]. MRI with contrast was done for further assessment using multi-sequence abdomen protocol. It was observed that the signal characteristics of the mass were similar to that of the spleen. In the arterial phase after gadovist injection, the pancreatic mass showed "zebra pattern" enhancement which is typical for the

This is an open access journal, and articles are distributed under the terms of the Creative Commons Attribution-NonCommercial-ShareAlike 4.0 License, which allows others to remix, tweak, and build upon the work non-commercially, as long as appropriate credit is given and the new creations are licensed under the identical terms.

For reprints contact: WKHLRPMedknow_reprints@wolterskluwer.com

Cite this article as: Yadav SP, Kotak SD, Kumar S, Pujari VS, ChandrasekaranA. Intrapancreatic accessory spleen: An imaging enigma. Indian J Radiol Imaging 2020;30:392-4.

Received: 22-May-2020 Revised: 16-Jul-2020 Accepted: 24-Jul-2020 Published: 15-Oct-2020 
spleen [Figure 2]. Diffusion weighted imaging was done at the $\mathrm{b}$ values of 0,400 , and $800 \mathrm{~s} / \mathrm{mm}^{2}$ and ADC map was generated. Six circular regions of interest (ROI) of the same size were drawn in the pancreatic mass, the spleen and normal pancreas in the ADC map and the mean ADC values were recorded [Figure 3]. In the next step, we used unpaired T-test on this data set (using SPSS version 19 for Microsoft Windows) and came to the conclusion that there was no significant difference between ADC values from the pancreatic mass and normal spleen $(P=0.753)$ while the ADC value of pancreatic mass differed significantly from that of normal pancreatic tissue $(P=0.0002)$. With these findings on CT and MRI, we inferred that the mass was an IPAS. Patient was followed up on MRI after 6 months and no change in the lesion was noted [Figure 4].

\section{Discussion}

An accessory spleen is a benign entity with its common locations near the splenic hilum and in the tail of the pancreas. Despite of its typical locations, confident radiological diagnosis warrants careful observation of its radiological appearance. This is critical as often an IPAS may be confused with pancreatic tumors like adenoma, carcinoma, and islet cell tumor. ${ }^{[3,5]}$ When a focal lesion within the pancreas shows similar signal characteristics and the same degree of enhancement as that of the spleen in all phases, possibility of an IPAS should be considered. ${ }^{[6,7]}$ Multiple imaging modalities like contrast-enhanced ultrasound and CT, superparamagnetic

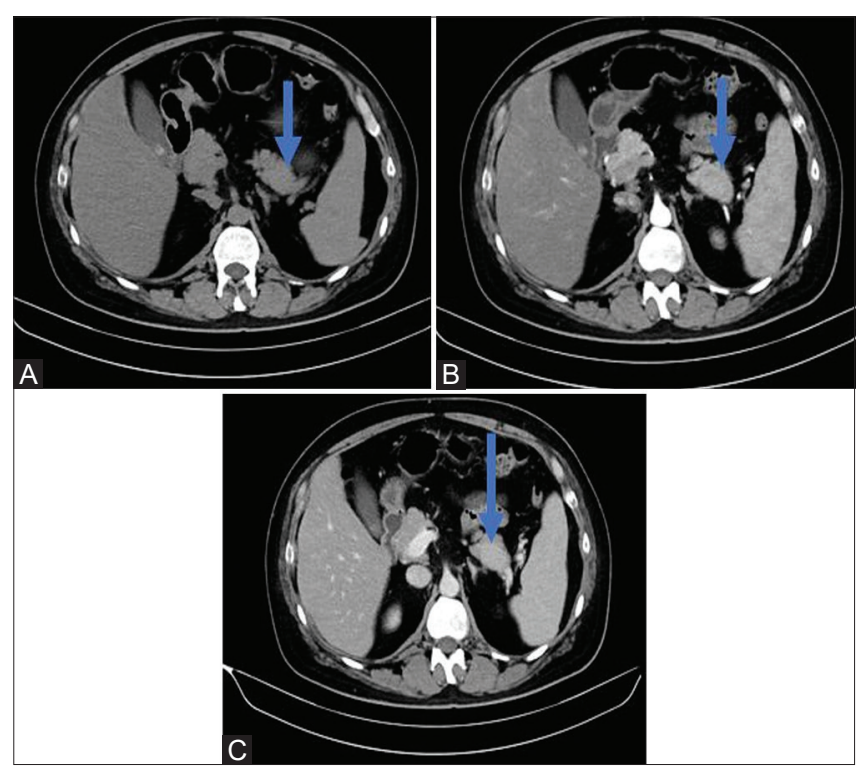

Figure $1(A-C)$ : (A) On precontrast axial CT image IPAS (arrow) is isodense compared to pancreas and spleen. (B) On contrast-enhanced axial CT image obtained during the arterial phase, the IPAS (arrow) is located in the tail of pancreas and it shows isoattenuation compared to the spleen. (C) On contrast-enhanced axial CT images obtained and portal venous phase IPAS (arrow) is located in the tail of pancreas and it shows isoattenuation compared to the spleen iron oxide-enhanced MR imaging, and nuclear medicine are available to distinguish IPAS from other types of pancreatic lesions. Among these, contrast-enhanced ultrasound, superparamagnetic iron oxide-enhanced MRI and nuclear medicine are very sensitive and specific for the diagnosis of IPAS, however they depend on phagocytosis of macrophages due to which the splenic visualization becomes difficult if there is minimal functioning of splenic tissue and more time may be required to acquire the essential images. ${ }^{[8]}$ Other drawbacks include lower spatial resolution of scintigraphy than CT or MRI, particularly in smaller lesions or lesions overlapping with the spleen. Ultrasound is operator dependent and has a limited sonic window to fully examine the pancreatic tail. ${ }^{[6,7,9]}$ In our case, the CT density and MR signals of the mass were equal to those of spleen before and after contrast administration. Also, the mass showed the same degree of enhancement as the spleen on postcontrast CT and MRI, including the typical zebra pattern of arterial phase enhancement. ${ }^{[10]}$ Ding et al. ${ }^{[11]}$ reported that no significant difference in the apparent diffusion coefficient was identified between the IPAS and orthotopic spleen $(P>0.05)$. Jang et al. ${ }^{[8]}$ in their study of 42 patients had found that small pseudopapillary tumor which is the principal differential diagnosis for IPAS differs significantly from the spleen in signal intensity on the diffusion-weighted and contrast-enhanced sequences while the IPAS shows the similar signal to that of the spleen. In agreement with the aforementioned studies, we observed that the mean ADC value of the pancreatic mass had no significant difference with that of the spleen

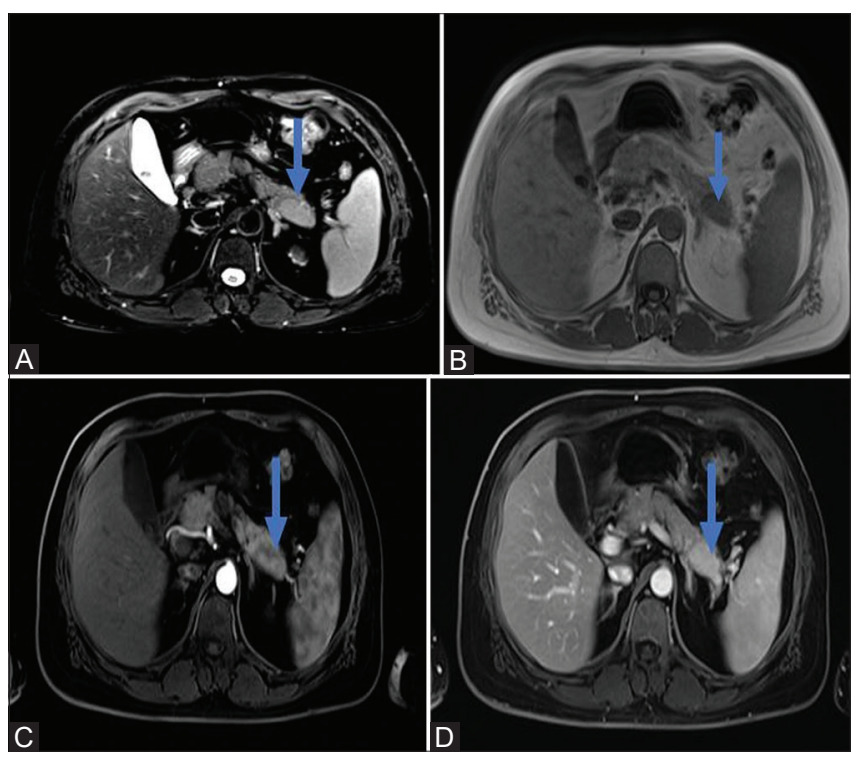

Figure 2 (A-D): (A) Fat saturated T2 weighted (SPACE) axial MRI image shows that the IPAS (arrow) is isointense to spleen while hyperintense than the pancreas. (B) T1 weighted axial MRI image shows isointensity of the IPAS (arrow) with orthotopic spleen and hypointensity with respect to the pancreas. (C) In the post-contrast arterial phase, the IPAS shows similar zebra pattern enhancement with that of the spleen. (D) In the post-contrast venous phase, the IPAS (arrow) shows similar enhancement with that of the spleen 


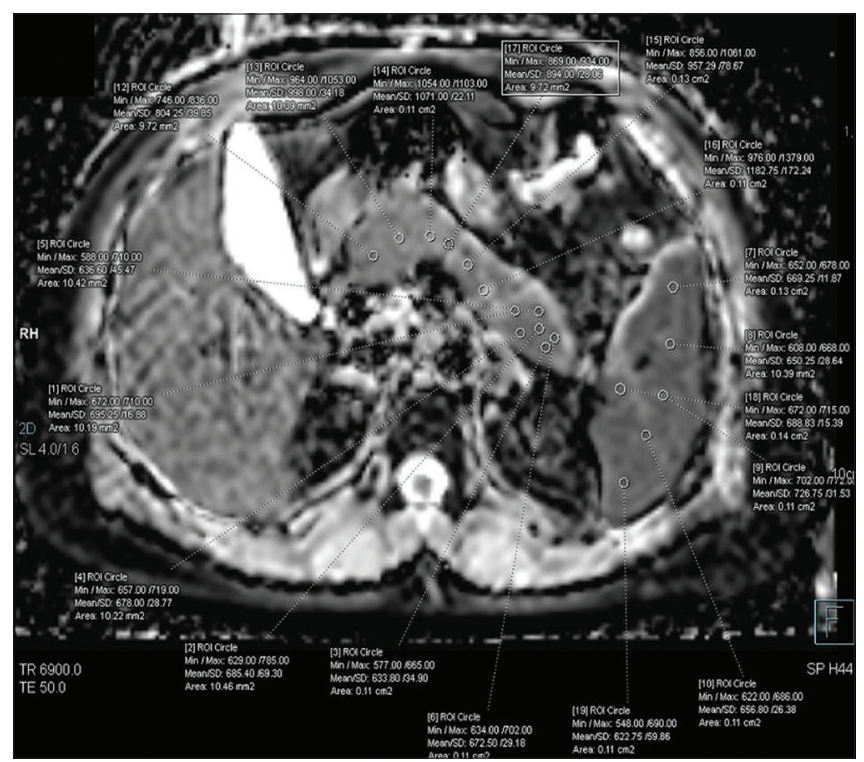

Figure 3: Axial ADC map generated after diffusion-weighted MRI showing the ROIs in the spleen, IPAS and the pancreas with respective $A D C$ values. No significant difference was found between the ADC values of IPAS and spleen $(P \geq 0.05)$, while a significant difference was there between the ADC values of IPAS and pancreas $(P \leq 0.05)$

while the ADC value of normal pancreatic parenchyma was much higher with a significant difference. Takayama et al. ${ }^{\left[{ }^{9]}\right.}$ suggested that to aid the clinical judgement, either biopsy or follow-up should be done to avoid unnecessary pancreatectomy. In this case, in view of the CT density and MR signals of the mass being equal to those of the spleen, the diagnostic possibility of an intrapancreatic accessory spleen was considered. Follow-up MRI was done after 6 months in which the lesion was stable.

In conclusion, the IPAS is a diagnosis which should be kept in mind as a differential while reviewing a case with pancreatic mass as it would prevent unnecessary intervention due to misdiagnosis of a pancreatic neoplasm. CT attenuation, MRI signal characteristics, post-contrast enhancement, and comparison of ADC values of the pancreatic mass with spleen and pancreas; all can lead to imaging diagnosis of IPAS, and the patient can be further followed up with imaging instead of immediate intervention in the form of biopsy or surgery.

\section{Declaration of patient consent}

The authors certify that they have obtained all appropriate patient consent forms. In the form the patient(s) has/have given his/her/their consent for his/her/their images and other clinical information to be reported in the journal. The patients understand that their names and initials will not be published and due efforts will be made to conceal their identity, but anonymity cannot be guaranteed.

Financial support and sponsorship

Nil.

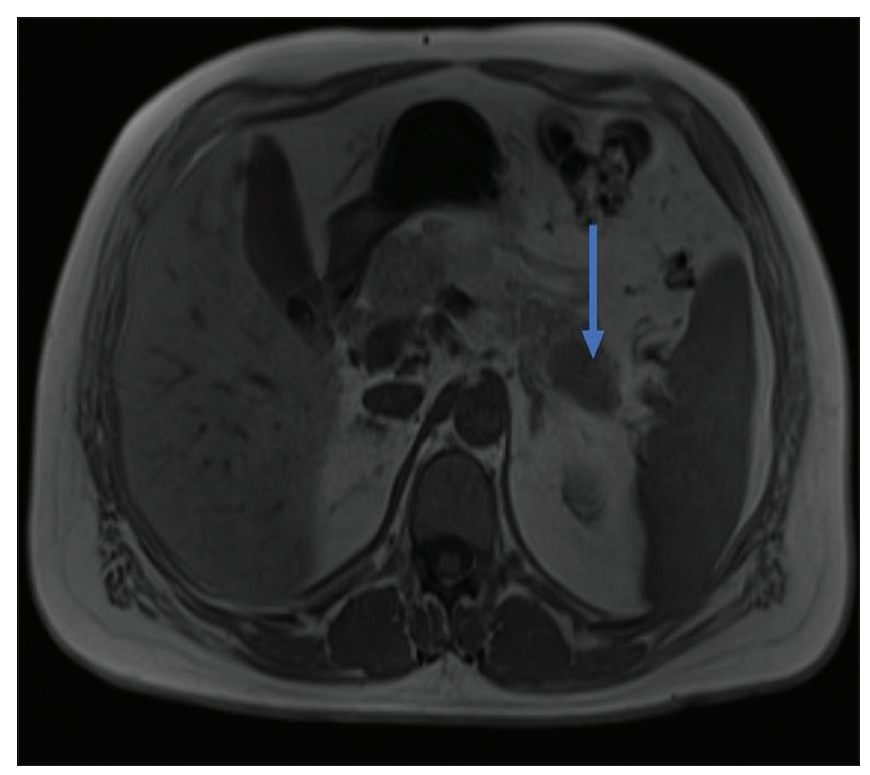

Figure 4: Follow up MRI after 6 months: T1 weighted axial image shows, the lesion (arrow) was still of the same size and signal intensity bolstering the diagnosis of IPAS

\section{Conflicts of interest}

There are no conflicts of interest.

\section{References}

1. Saunders TA, Miller TR, Khanafshar E. Intrapancreatic accessory spleen: Utilization of fine needle aspiration for diagnosis of a potential mimic of a pancreatic neoplasm. J Gastrointest Oncol 2016;7:S62-5.

2. Guo W, Han W, Liu J, Jin L, Li J-S, Zhang Z-T, et al. Intrapancreatic accessory spleen: A case report and review of the literature. World J Gastroenterol 2009;15:1141-3.

3. Rosenkranz W, Kamhi B, Horowitz M. Retroperitoneal accessory spleen simulating a suprarenal mass. Br J Radiol 1969;42:939-42.

4. Matthaei H, Schmelzle M, Braunstein S, Bölke E, Peiper M. Pancreatic incidentalomas: A growing clinical challenge exemplified by an intrapancreatic accessory spleen. Wien Klin Wochenschr 2011;123:186-8.

5. Churei $H$, Inoue $H$, Nakajo M. Intrapancreatic accessory spleen: Case report. Abdom Imaging 1998;23:191-3.

6. Hayward I, Mindelzun RE, Jeffrey RB. Intrapancreatic accessory spleen mimicking pancreatic mass on CT. J Comput Assist Tomogr 1992;16:984-5.

7. Harris GN, Kase DJ, Bradnock H, Mckinley MJ. Accessory spleen causing a mass in the tail of the pancreas: MR imaging findings. AJR Am J Roentgenol 1994;163:1120-1.

8. Jang KM, Kim SH, Lee SJ, Park MJ, Lee MH, Choi D. Differentiation of an intrapancreatic accessory spleen from a small $(<3-\mathrm{cm})$ solid pancreatic tumor: Value of diffusion-weighted MR imaging. Radiology 2013;266:159-67.

9. Takayama T, Shimada K, Inoue K, Wakao F, Yamamoto J, Kosuge T. Intrapancreatic accessory spleen. Lancet 1994;344:957-8.

10. Giambelluca D. The “zebra spleen.” Abdom Radiol 2019;44:1189-90.

11. Ding Q, Ren Z, Wang J, Ma X, Zhang J, Sun G, et al. Intrapancreatic accessory spleen: Evaluation with CT and MRI. Exp Ther Med 2018;16:3623-31. 